\title{
Identification and characterization of wheat long non-protein coding RNAs responsive to powdery mildew infection and heat stress by using microarray analysis and SBS sequencing
}

Mingming Xin ${ }^{1,2+}$, Yu Wang ${ }^{1,2+}$, Yingyin Yao ${ }^{1,2+}$, Na Song ${ }^{1,2}$, Zhaorong Hu ${ }^{1,2}$, Dandan Qin ${ }^{1,2}$, Chaojie Xie ${ }^{1,2}$, Huiru Peng ${ }^{1,2^{*}}$, Zhongfu $\mathrm{Ni}^{1,2}$ and Qixin Sun ${ }^{1,2,3^{*}}$

\begin{abstract}
Background: Biotic and abiotic stresses, such as powdery mildew infection and high temperature, are important limiting factors for yield and grain quality in wheat production. Emerging evidences suggest that long non-protein coding RNAs (npcRNAs) are developmentally regulated and play roles in development and stress responses of plants. However, identification of long npcRNAs is limited to a few plant species, such as Arabidopsis, rice and maize, no systematic identification of long npcRNAs and their responses to abiotic and biotic stresses is reported in wheat.

Results: In this study, by using computational analysis and experimental approach we identified 125 putative wheat stress responsive long npcRNAs, which are not conserved among plant species. Among them, some were precursors of small RNAs such as microRNAs and siRNAs, two long npcRNAs were identified as signal recognition particle (SRP) 7S RNA variants, and three were characterized as U3 snoRNAs. We found that wheat long npcRNAs showed tissue dependent expression patterns and were responsive to powdery mildew infection and heat stress.

Conclusion: Our results indicated that diverse sets of wheat long npcRNAs were responsive to powdery mildew infection and heat stress, and could function in wheat responses to both biotic and abiotic stresses, which provided a starting point to understand their functions and regulatory mechanisms in the future.
\end{abstract}

\section{Background}

The developmental and physiological complexity of eukaryotes could not be explained solely by the number of protein-coding genes [1]. For example, the Drosophila melanogaster genome contains only twice as many genes as some bacterial species, although the former is far more complex in its genome organization than the latter. Similarly, the number of protein-coding genes in human and nematode is extremely close. A portion of this paradox can be resolved through alternative pre-

\footnotetext{
* Correspondence: penghuiru@cau.edu.cn; qxsun@cau.edu.cn

+ Contributed equally

${ }^{1}$ State Key Laboratory for Agrobiotechnology and Key Laboratory of Crop Heterosis and Utilization (MOE) and Key Laboratory of Crop Genomics and Genetic Improvement (MOA), Beijing Key Laboratory of Crop Genetic Improvement, China Agricultural University, Beijing, 100094, PR China Full list of author information is available at the end of the article
}

mRNA splicing [2]. In addition, post-translational modifications can also contribute to the increased complexity and diversity of protein species [3].

Recent studies suggest that most of the genome are transcribed, among the transcripts only a small portion encode for proteins, whereas a large portion of the transcripts do not encode any proteins, which are generally termed non-protein coding RNAs (npcRNA). For example, transcriptome profiling in rice (Oryza sativa) indicates that there are about 8400 putative npcRNAs, which do not overlap with any predicted open reading frames (ORFs) [4]. These npcRNAs are subdivided as housekeeping npcRNAs (such as transfer and ribosomal RNAs) and regulatory npcRNAs or riboregulators, with the latter being further divided into short regulatory npcRNAs $(<300$ bp in length, such as microRNA, siRNA, piwi-RNA) and long regulatory npcRNAs 
(>300 bp in length). With the identification of microRNAs and siRNAs in diverse organisms, increasing evidences indicate that these short npcRNAs play important roles in development, responses to biotic and abiotic stresses by cleavage of target mRNAs or by interfering with translation of target genes [5-9].

Long npcRNAs are transcribed by RNA polymerase II, polyadenylated and often spliced [10]. Studies in mice and human suggested that at least $13 \%$ and $26 \%$ of the unique full-length cDNAs, respectively, are thought to be poly(A) tail-containing long npcRNAs [11-13]. Emerging evidences also suggest that long npcRNAs are developmentally regulated and responsive to external stimuli, and play roles in development and stress responses of plants and disease in human. For example, some long npcRNAs are regulated in various stresses in plants and animals [9,14-16]. In Caenorhabditis elegans, 25 npcRNAs are either over- or under-expressed under heat shock or starvation conditions [17], while in Arabidopsis, the abundance of 22 putative long npcRNAs are regulated by phosphate starvation, salt stress or water stress [18]. In Arabidopsis, long npcRNA, COOLAIR (cold induced long antisense intragenic RNA), is coldinduced FLC antisense transcripts, and has an early role in the epigenetic silencing of FLC and to silence FLC transcription transiently [19]. Long npcRNA HOTAIR in human is reported to reprogram chromatin state to promote cancer metastasis [20].

Currently, two computational methods are employed to identify long npcRNAs, genome-based and transcriptbased. Using genomic sequences, more than 200 candidate long npcRNAs were predicted in Escherichia coli [21], and at least 20 long npcRNA genes have been experimentally confirmed [22]. In Rhizobium etli, 89 candidate npcRNAs are detected by high-resolution tilling array, and 66 are classified as novel ones [23]. While using cDNA or EST sequences, a large number of long npcRNAs are detected in Drosophila, mouse and Arabidopsis [12,18,24-26].

Up to date, identification of long npcRNAs is limited to a few plant species, such as Arabidopsis, rice and maize. To our best knowleage, in wheat no systematic identification of long npcRNAs is reported. Wheat (Triticum aestivum, AABBDD, $2 \mathrm{n}=42$ ) is the most widely grown crop plant, occupying $17 \%$ of all the cultivated land, provides approximately $55 \%$ of carbohydrates for world human consumption [27], Biotic and abiotic stresses are important limiting factors for yield and grain quality in wheat production. For instance, powdery mildew, caused by the obligate biotrophic fungus Blumeria graminis f. sp. tritici (Bgt), is one of the most devastating diseases of wheat in China and worldwide and causing significant yield losses [28]. High temperature, often combined with drought stress, causes yield loss and reduces the grain quality [29]. To reduce the damages caused by biotic and abiotic stresses, plants have evolved sophisticated adaptive response mechanisms to reprogram gene expression at the transcriptional, posttranscriptional and post-translational levels [30]. Recently, transcript profiling has been successfully employed to determine the transcriptional responses to powdery mildew infection and heat stress in wheat, and the results revealed that a number of genes were significantly induced or repressed in response to these stresses [31,32].

In our previous study [33], it was demonstrated that expression of microRNAs in wheat was regulated by powdery mildew infection and heat stress, which stimulated us to explore whether long npcRNA was also responsive to powdery mildew infection and/or heat stress. In this study, we performed a genome-wide in silico screening of powdery mildew infection and heat stress responsive wheat transcripts in order to isolate a collection of long npcRNA genes. Combining microarray analysis and high-throughput SBS sequencing methods, we totally characterized 125 putative stress responsive long npcRNAs in wheat, four of them were miRNA precursors, and one was experimentally verified by northern blot. Wheat long npcRNAs displayed tissue-specific expression patterns and their expression levels were altered in response to powdery mildew infection and/or heat stress, which suggested that at least a subset of these newly identified wheat long npcRNAs potentially play roles in response to biotic and/or abiotic stresses in wheat.

\section{Results}

Identification of powdery mildew infection and heat stress responsive long npcRNA candidates in wheat In our previous study, a total of 9744 powdery mildew infection and 6560 heat stress responsive transcripts were obtained (with a fold change of at least 2) through microarray analysis using the wheat Affymetrix GeneChip $^{\circledR}$. In this study, in order to identify the putative wheat long npcRNAs which were responsive to powdery mildew and/or heat stress, these stress responsive transcripts were used to characterize the wheat long npcRNAs. Firstly, these transcripts were annotated by Harvest program, and 7746 and 5754 transcripts were identified to be protein-coding genes and therefore were discarded in further analysis. The remaining transcripts were then analyzed by Blastx and Blastn, 586 and 406 ESTs with no similarity to protein coding genes or tRNA and rRNA were retained. Secondly, 125 transcripts with no or short ORFs (less than 80aa) and polyA-tails were selected as putative long npcRNAs (Additional file 1), among which 71 were responsive to powdery mildew infection, and 77 were responsive to 
heat stress. We found that 23 long npcRNAs responded to both powdery mildew infection and heat stress (designated TalnRNA). A total of 48 putative long npcRNAs were only responsive to powdery mildew infection (designated TapmlnRNA), and 54 were only responsive to heat stress (designated TahlnRNA). Among these putative long npcRNAs, the longest ORF was 74aa, with an average of 43.5aa (Additional file 1). In order to validate expression patterns of the long npcRNAs in response to powdery mildew infection and/ or heat stress, expression patterns of 4 long npcRNAs, TapmlnRNA19, TapmlnRNA30, TahlnRNA27 and TalnRNA5, were determined by using quantitative RTPCR analysis. Expression levels of TapmlnRNA19 and TapmlnRNA30 were up-regulated after powdery mildew inoculation (Figure 1a, b), whereas expression of TahlnRNA27 and TalnRNA5 were up-regulated after heat stress (Figure 2a, b), which showed consistent expression patterns with microarray analysis.

\section{Four long npcRNA transcripts correspond to miRNA precursors}

By mapping miRNAs which were identified from our previously sequenced six small RNA libraries (S-0h, S-12h, R-0h, R-12h, TAM-0h, TAM-1h) [33] to the complete collection of 125 long npcRNAs, we identified that four transcripts (TalnRNA5, TapmlnRNA8, TapmlnRNA19, TahlnRNA27) were miRNA precursors. Prediction of the secondary structure for the four transcripts by using the Vienna RNA package RNAfold web interface program showed that these four miRNA precursors had stable hairpin structures (Additional file 2, 3, 4 and 5).

Among the four long npcRNAs, three (TalnRNA5, TapmlnRNA19 and TapmlnRNA8) were responsive to powdery mildew infection. Both TalnRNA5 and TapmlnRNA19 were the precursors of miR2004, and TapmlnRNA8 was the precursor of miR2066. It is interesting to note that TapmlnRNA19 and TalnRNA5 were up-regulated after powdery mildew infection as determined by qRT-PCR (Figure 1a, 3a), and miR2004 was

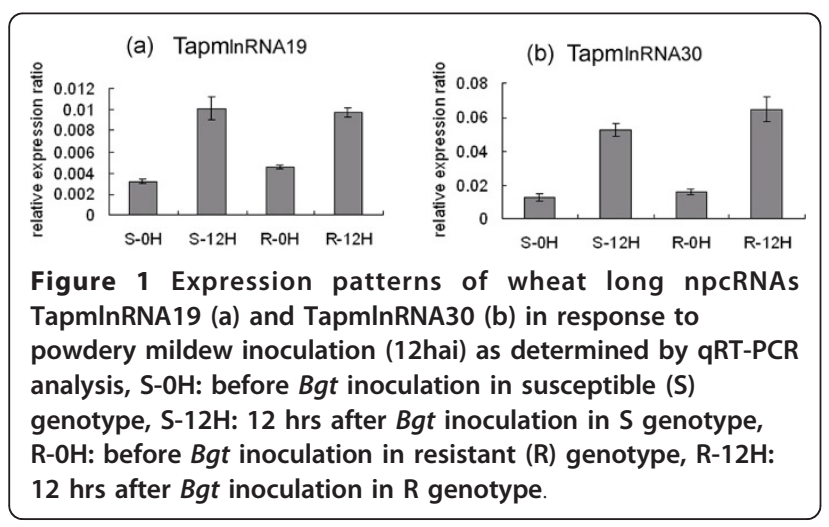

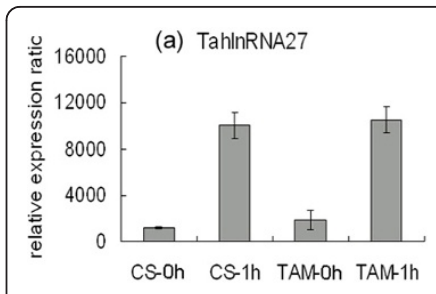

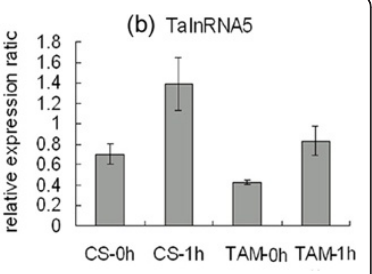

Figure 2 Expression patterns of wheat long npcRNAs TahInRNA27 (a) and TalnRNA5 (b) in response to heat stress. CS-Oh: before heat stress treatment for heat susceptible genotype Chinese Spring (CS), CS-1h: after 1 hour heat stress treatment, TAMOh: before heat stress treatment for heat tolerant genotype TAM107 (TAM), TAM-1h: after 1 hour heat stress treatment.

also found to be up-regulated based on the small RNA high throughput sequencing (Figure $3 \mathrm{~b}$ ). To further determine the expression pattern of miR2004, we performed Northern blot analysis (Figure 3c) which indicated that miR2004 shared similar expression pattern with the high throughput sequencing.

The heat responsive long npcRNA TahlnRNA27 contained Ta-miR2010 family sequences, and was upregulated in 'TAM107' (heat tolerant cultivar) $1 \mathrm{~h}$ after heat treatment (Figure 2a), whereas Ta-miR2010 was also statistically up-regulated $1 \mathrm{~h}$ after heat stress in the small RNA databases of 'TAM107' in our previous study [33]. The secondary structure and the corresponding expression pattern indicated that TahlnRNA27 might be the precursor of miR2010. In addition, the powdery mildew infection responsive long npcRNA TalnRNA5 (Figure 3a) was found to be also responsive to heat stress and the expression level was increased in 'CS' and 'TAM107' $1 \mathrm{~h}$ after heat stress (Figure 2b).

\section{Characterization of putative long npcRNAs for siRNA}

We found that 16 out of 71 powdery mildew responsive long npcRNAs gave rise to small RNAs (Additional file 1 ), and all of them had similar expression pattern in microarray analysis and SBS sequencing. Most of these long npcRNAs produced more than one small RNA family. For example, TapmlnRNA11 comprised three small RNA family sequences and each had several members (Figure 4). The expression level of TapmlnRNA11 in non-inoculated genotypes was quite low, but accumulated to a high level after powdery mildew infection in JD8 and JD8-Pm30 12hai (Figure 5a). Consistent with this expression pattern, its corresponding siRNAs were also up-regulated after powdery mildew infection (Figure $5 b)$ in both genotypes.

For the heat stress responsive long npcRNAs, there were nine transcripts matching the small RNAs (Additional file 1). Among them, TalnRNA21 was responsive to both heat treated and powdery mildew inoculated wheat leaves, however, the expression pattern was quite 
(a) TalnRNA5

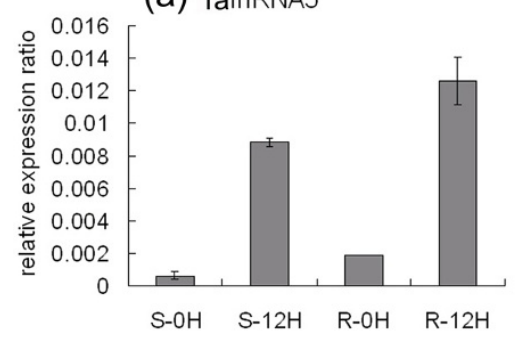

(c)

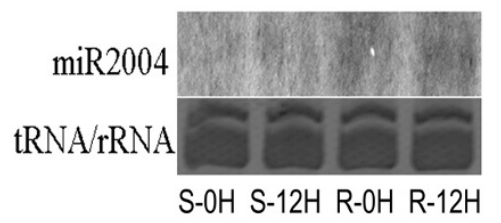

(b) $\operatorname{miR2004}$

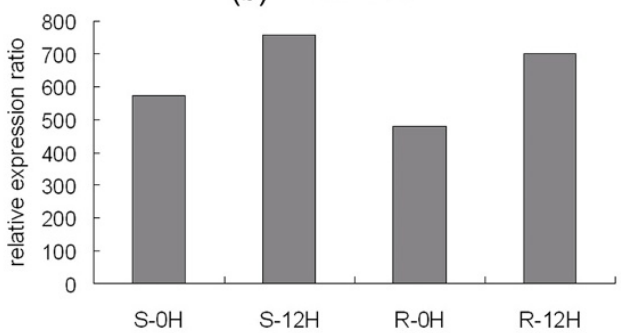

Expression pattern of wheat long npcRNA TalnRNA5 and its corresponding miRNA before or $12 \mathrm{hai}$ in both disease resistant genotype (R) and susceptible genotype (S). (a) The expression level of TalnRNA5 as determined by qRT-PCR. (b) The expression pattern of miR2004 based on high throughput sequencing. (c) Northern blot analysis for miR2004 expression before or 12 hai in $\mathrm{S}$ genotype and R genotype.

different, expression of TalnRNA21 was repressed in JD8 and JD8-Pm30 12hai (Figure 6a), but up-regulated after heat stress in 'CS' and 'TAM107' (Figure 6b). We also noted that TalnRNA21 accumulated to a much higher expression level $1 \mathrm{~h}$ after heat treatment in heat tolerant cultivar as compared to that in heat sensitive cultivar (Figure 6b).

\section{Long npcRNAs corresponding to SRP and snoRNAs}

We found that 52 powdery mildew infection responsive and 66 heat stress responsive long npcRNAs could execute their functions in the form of long molecules, among which 21 transcripts were responsive to both stress treatments (Additional file 1). Two transcripts, TalnRNA9 and TalnRNA12, were identified as signal recognition particle (SRP) 7S RNA variant 1 and 3, respectively. It was found that the expression of TalnRNA9 was increased in both JD8 and JD8-Pm30 genotypes 12 hours after infection (hai) (Figure 7a), but was repressed $1 \mathrm{~h}$ after heat treatment in 'CS' (heat sensitive cultivar) and 'TAM107' (heat tolerant cultivar) (Figure $7 \mathrm{~b}$ ). Among the 45 long npcRNAs which were only responsive to heat stress, three (TahlnRNA12 TahlnRNA23 and TahlnRNA29) were characterized as U3 snoRNAs, and their expression levels were increased $1 \mathrm{~h}$ after heat stress in both 'CS' and 'TAM107'(Figure 8)

\section{Histone acetylation of TalnRNA5 and TapmInRNA19}

The histone acetylation levels of TalnRNA5 and TapmlnRNA19 were detected using antibody H3K 9 by ChIP according to the procedure of Lawrence [34]. ChIP analysis indicated that acetylation levels of
TalnRNA5 and TapmlnRNA19 in the inoculated JD8 and JD8-Pm30 increased as compared to the noninoculated controls (Figure 9).

\section{Small RNAs might influence long npcRNAs expression}

Based on our analysis, two SRP 7S RNA variants TalnRNA 9 and TalnRNA12 could be regulated by $24 \mathrm{nt}$ siRNAs. There were five siRNA families complementarily matching to the long npcRNAs, among which, three groups (group I, group II, group III) matched both TalnRNA9 and TalnRNA12, and other two (group IV group V) were specific for TalnRNA9 (Additional file 6). We designed gene specific primers (Additional file 7) and amplified the antisense strand sequences of TalnRNA9 and TalnRNA12 (anti-TalnRNA9 and anti- TalnRNA12). It was found that expression levels of TalnRNA9 and TalnRNA12 were up-regulated after powdery mildew inoculation in the two genotypes (Figure 10a), whereas both of the antisense sequences were down-regulated after powdery mildew inoculation in the two genotypes (Figure 10b), and negative correlation in expression levels was observed between sense strand and antisense strand expression patterns in both JD8 and JD8-Pm30 (Figure 10). In addition, three long npcRNAs, TapmlnRNA11, TapmlnRNA41 and TapmlnRNA42 also had several group small sequences matching them, and their expression patterns could be also regulated by siRNAs.

\section{Wheat putative long npcRNAs displayed tissue-specific expression patterns}

To investigate the expression patterns of long npcRNAs in different wheat tissues, qRT-PCR was performed in 8 


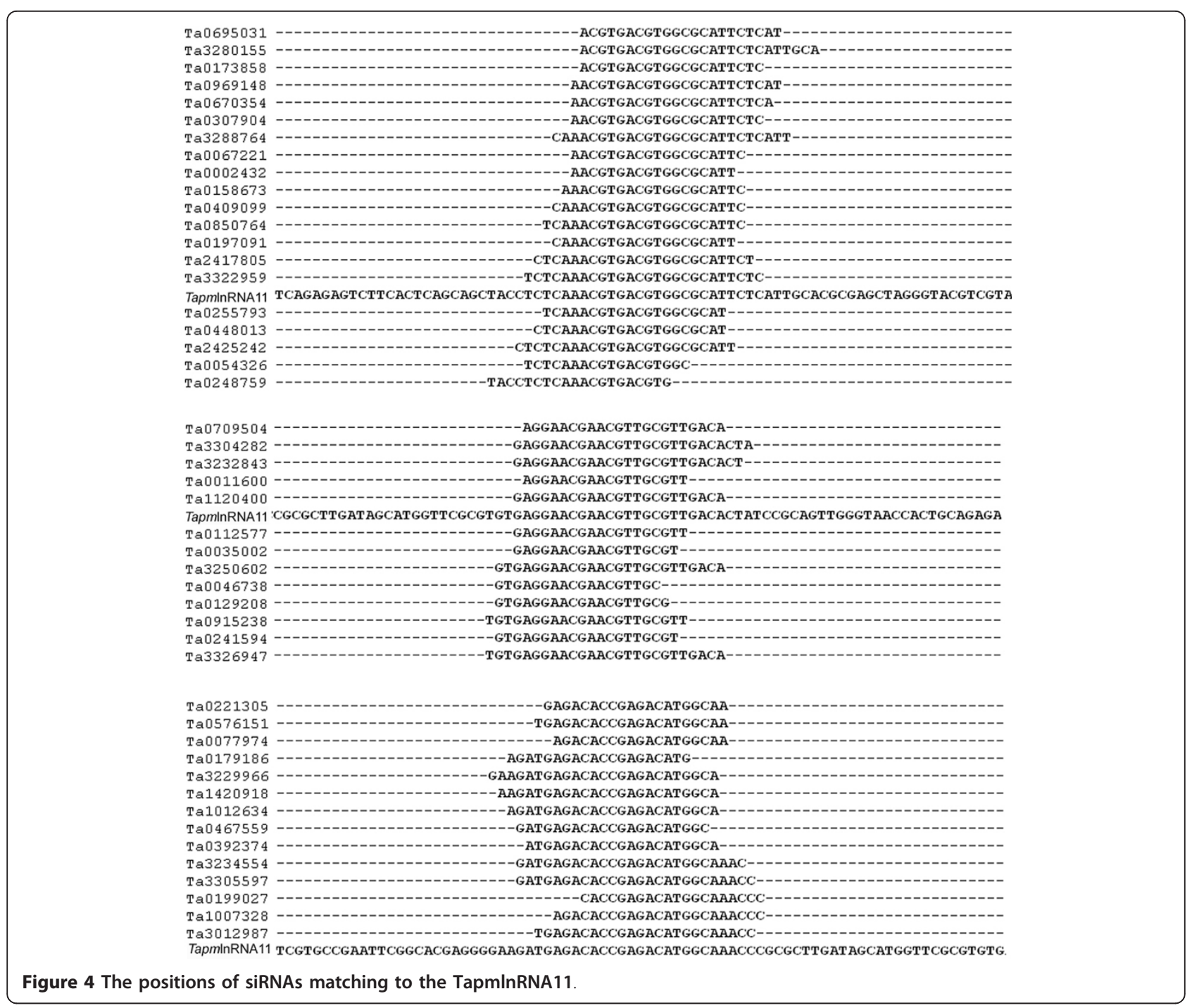

wheat tissues using gene specific primer pairs (Additional file 7), including leaf, internode, flag leaf, root, seed, awn, young spike and glume (Figure 11).

It was found that wheat long npcRNAs displayed tissue-specific expression patterns. TapmlnRNA30 was only detected in seed, whereas TapmlnRNA19 accumulated preferentially in young spike (Figure 11). TalnRNA5 was expressed in all the tissues, but expression level was relatively higher in seed as compared to other tissues (Figure 11). TalnRNA9 was abundantly

\section{(a) TapmInRNA11}

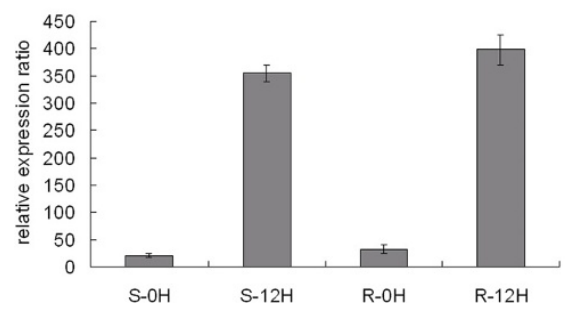

(b) siRNAs corresponding toTapmInRNA11

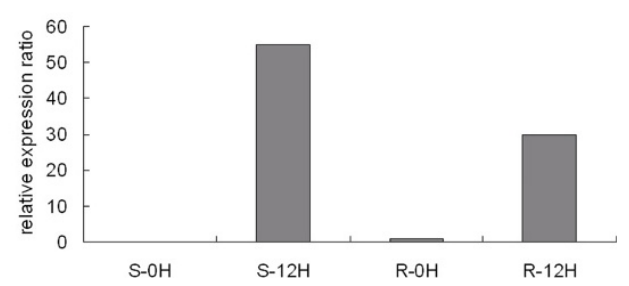

Figure 5 Expression patterns of wheat long npcRNAs and their corresponding siRNAs before or 12 hai in $\mathrm{S}$ genotype and R genotype. (a) The expression pattern of TapmlnRNA11 in wheat microarray analysis. (b) The abundance of corresponding siRNAs matching TapmlnRNA11 based on high-throughput sequencing. 

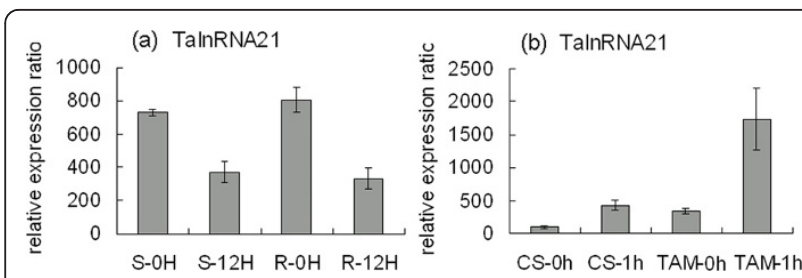

Figure 6 The expression pattern of TalnRNA21 in response to powdery mildew inoculation (a) and heat stress (b) based on microarray analysis.

expressed in leaf, root and seed, no signal was detected in other tissues (Figure 11). Interestingly, although both TalnRNA5 and TapmlnRNA19 gave rise to miR2004, their expression patterns were obviously different (Figure 11). In addition, TalnRNA9 was expressed quite differently between leaf and flag leaf, and the transcripts accumulated predominantly in leaf (Figure 11).

\section{Experimentally verified full length CDNA of predicted long npcRNAs}

In order to obtain the full length cDNAs corresponding to the long npcRNAs, we performed 5'RACE for four long npcRNAs, including TapmlnRNA26, TalnRNA21, TahlnRNA37 and TahlnRNA47. The cDNA from young leaf of JD8 was amplified by using gene specific primers (Additional file 7) and sequenced. The full length cDNAs corresponding to TapmlnRNA26, TalnRNA21, TahlnRNA37 and TahlnRNA47 were 1599 bp, 1497 bp, $737 \mathrm{bp}$ and $988 \mathrm{bp}$ in length, respectively. The ORFs of these sequences were searched by using ORF finder program, and no ORFs longer than 80aa was found in these full length cDNAs (Additional files 8, 9 and 10). For example, TapmlnRNA26 contained 15 putative ORFs, but the longest ORF was only 74aa (Figure 12).

\section{Discussion}

Wheat long npcRNAs are not conserved among the plant species and responsive to both biotic and abiotic stresses By using combination of microarray and SBS sequencing, a total of 125 putative long npcRNAs were identified in wheat leaves using strict criteria across a collection of more than 9700 powdery mildew and 6500
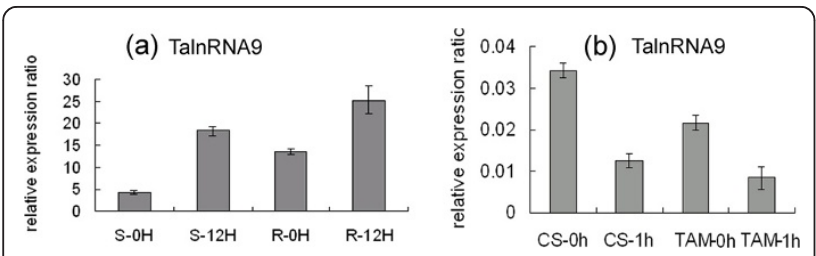

Figure 7 The expression patterns of TalnRNA9 in response to powdery mildew inoculation (a) and heat stress (b) as determined by qRT-PCR. heat stress responsive sequences. Our analysis could fail to identify the bona fide long npcRNAs in wheat due to the limited genomic information and gene annotation of wheat, however, these 125 putative long npcRNAs constituted a reliable set of wheat long npcRNAs. It must be pointed out that, in the absence of wheat whole genomic information and the full length sequences of these wheat long npcRNAs, some of them might turn out to be protein-coding RNAs when the wheat genomic sequences are available. However, this study represents the first attempt to characterize the wheat long npcRNAs and their responses to biotic and/or abiotic stresses, which could provide a starting point for further investigation of long npcRNAs in wheat.

As most non-protein coding RNAs were subjected to a low degree of evolutionary constraint, we found that the 125 long npcRNAs identified in this study had no homologs or significant matches out of plant, animal and microorganism kingdoms, and were wheat specific except for two SRP 7SRNA variants (TalnRNA9 and TalnRNA12) and 3 U3 snoRNAs (TahlnRNA12 TahlnRNA23 and TahlnRNA29), which was in good agreement to the previous studies in other species such as Drosophila, Arabidopsis and mouse [12,25,26]. Also, these long npcRNAs did not appear to form large homologous family. This might suggest that during the evolution, wheat had developed a batch of specific long npcRNAs to regulate gene expression and cell activity. Further analysis revealed that long npcRNAs in wheat had tissue-specific expression patterns, similar expression patterns of long npcRNAs were also reported in other species [24-26]. In our investigation, even in leaf and flag leaf, TalnRNA9 was differentially expressed, which suggested that long npcRNAs probably had much more precise expression regulation mechanisms. In addition, though TalnRNA5 and TapmlnRNA19 gave rise to the same miRNA, they displayed distinct expression patterns, indicating that miRNA could potentially be produced by different precursors in different wheat tissues.

SRP RNA is an exception, as it is a ribonucleoprotein (protein-RNA complex) that recognizes and targets specific proteins to the endoplasmic reticulum in eukaryotes and the plasma membrane in prokaryotes. Moreover, U3 snoRNAs predominantly found in the nucleolus are thought to guide site-specific cleavage of ribosomal RNA (rRNA) during pre-rRNA processing. Therefore, they are thought to be conserved across three kingdoms.

It was reported that increased expression of either BC200 or an antisense transcript of the b-secretase-1 (BACE1) gene had been implicated in the progression of Alzheimer's disease [35,36]. Ben et al [18] found that abiotic stress altered the accumulation of 22 out of the 76 npcRNAs. These indicated that long npcRNAs had 

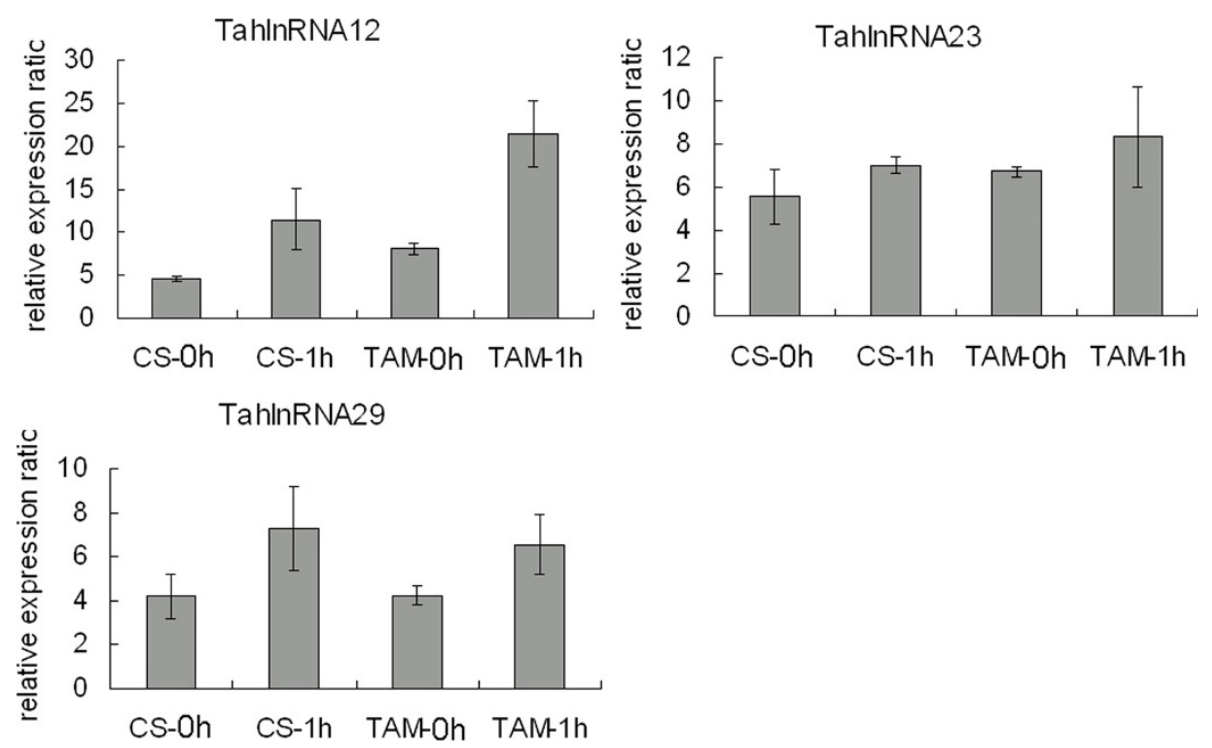

Figure 8 The expression patterns of TahInRNA12, TahlnRNA23 and TahlnRNA29 $1 \mathrm{~h}$ after heat stress in heat sensitive genotype ('CS') and heat tolerant genotype ('TAM107') based on microarray analysis.

been linked to biotic and/or abiotic stresses, though in most instances, evidence had relied on differences in transcript expression levels between treated and nontreated samples. Our analysis added further evidence for the responsiveness of long npcRNAs to both biotic and/ or abiotic stresses, since 71 wheat long npcRNAs were responsive in defense against powdery mildew infection, and 77 were responsive to heat stress.

\section{Some of the wheat long npcRNAs are small RNA precursors}

Study showed that miR675 was derived from the long npcRNA H19 which was endogenously expressed in human keratinocytes and neonatal mice [37], and . npcRNA78 gene contained the miR162 sequence in an alternative intron and corresponded to the MIR162a locus [24]. The 'BIC' noncoding RNA that served as the precursor for miR155 was also readily detectable in vivo as fulllength transcripts [38]. In our identified wheat long npcRNAs, four transcripts (TalnRNA5, TapmlnRNA8, TapmlnRNA19, and TahlnRNA27) were characterized as putative miRNA precursors. Among them, TapmlnRNA8,

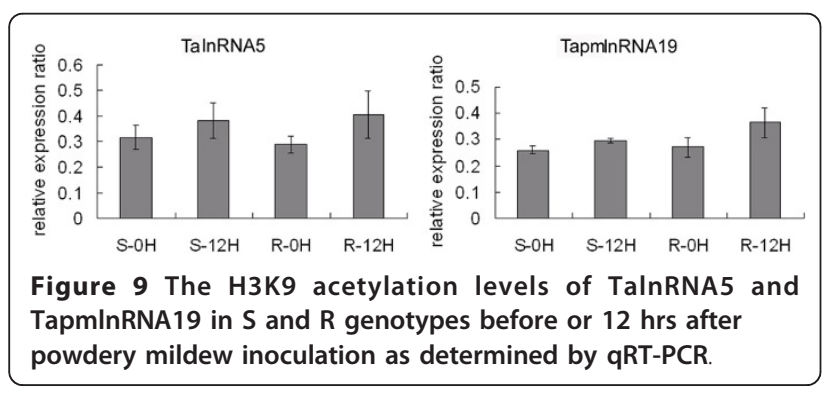

TapmlnRNA19 were specific to powdery mildew infection, while TahlnRNA27 was only responsive to heat stress. Increasing evidence indicated that miRNAs played important roles in plant responses to biotic stresses $[9,39,40]$. After powdery mildew infection, TalnRNA5 and TapmlnRNA19 were up-regulated 12hai in JD8 and JD8Pm30 genotypes, and their corresponding miR2004 was also increased in abundance, which strongly indicated that these two long npcRNAs were processed to miRNAs to regulate wheat response to powdery mildew infection. However, as there were no significant expression differences between the NILs JD8 and JD8-Pm30, we speculated that these two long npcRNAs functioned as basal defense. To further confirm this hypothesis, TalnRNA5, TapmlnRNA19, TalnRNA9 and TapmlnRNA30 were analyzed using qRT-PCR in $12 \mathrm{hrs}$ after-touched JD8 and JD8-Pm30 as well as their controls, and their expression level had no differences between two treatments (data not show), which suggested that the expression alteration were caused by powdery mildew infection, not by touching.

In addition, we revealed that 26 wheat long npcRNAs produced siRNAs and 97 sequences could function in the form of long molecules involved in wheat resistance to powdery mildew infection and/or heat stressed. The collection of long npcRNAs offered candidates for further analysis of this kind of npcRNAs, which gained increasing attention in recent years $[9,41,42]$.

Our analysis revealed that two SRP 7S RNA variants (TalnRNA9 and TalnRNA12) as well as TapmlnRNA11, TapmlnRNA41 and TapmlnRNA42 could be regulated by siRNAs. Coram et al [43] reported that the antisense strands of probe sets Ta.21480 and Ta.24771 (namely, 

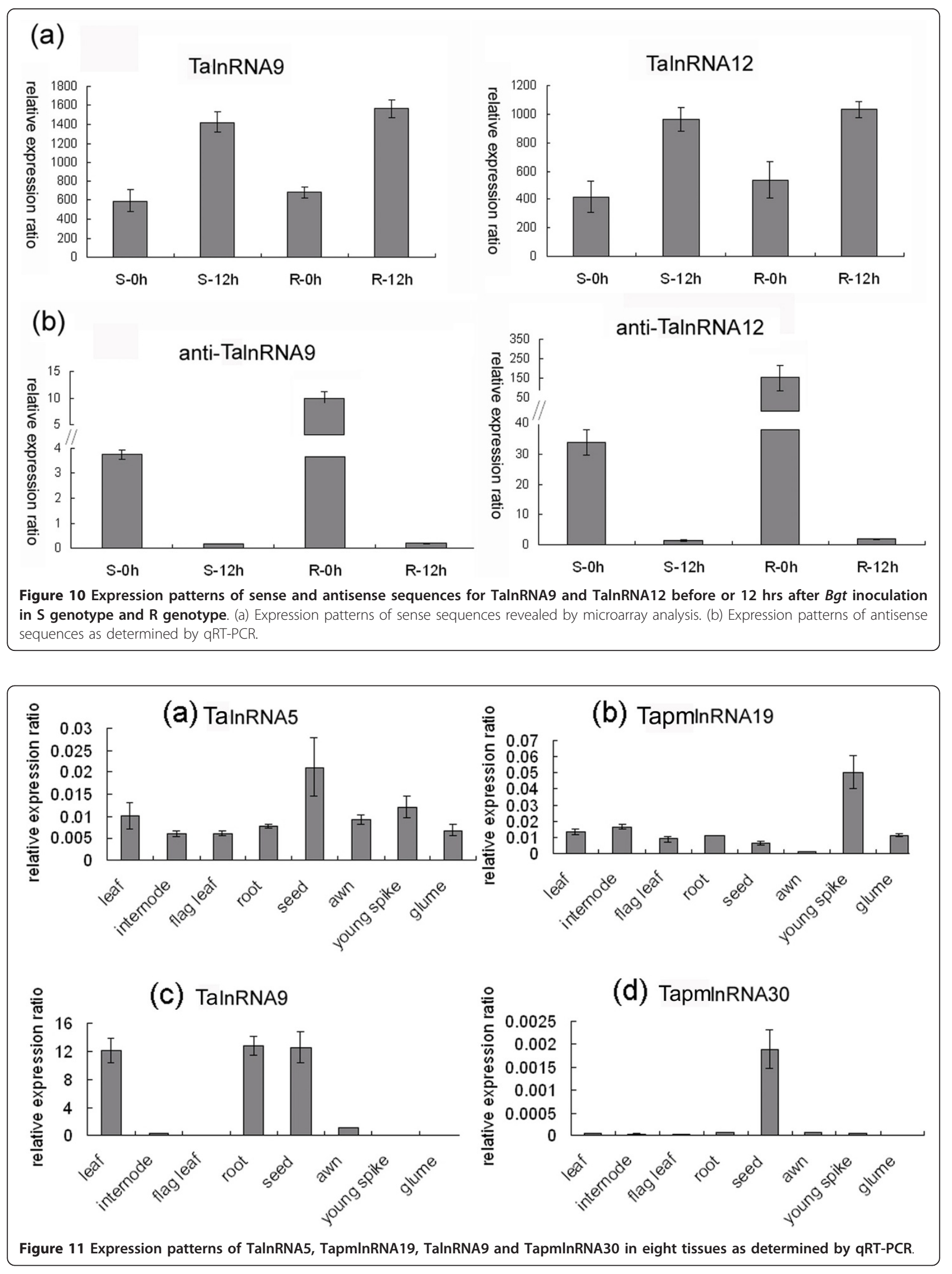


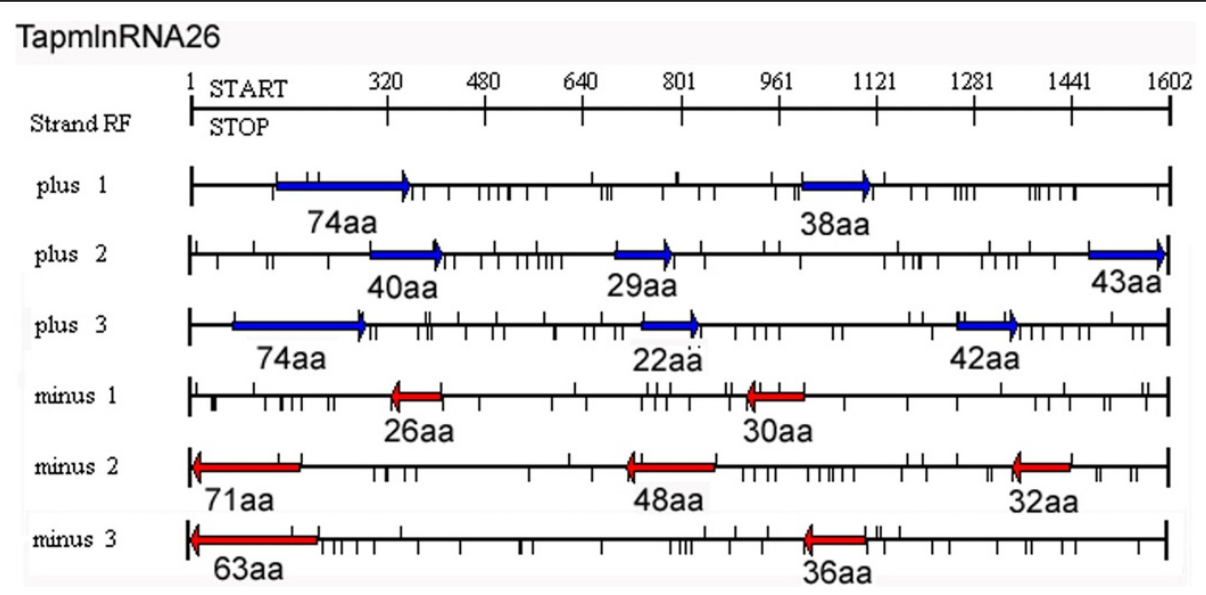

Figure 12 The 15 short possible open reading frames (ORFs) positioned in TapmInRNA26.

TalnRNA9 and TalnRNA12) were expressed using wheat Affymetrix genome array, which was in good agreement with our experimental results. And interestingly, our analysis also shown that the expression patterns of antisense had negative correlations with sense sequence for both TalnRNA9 and TalnRNA12, which strongly indicated that the siRNAs generated from antisense strands might regulate expression of their corresponding sense strands. Collectively, this study indicated that expression of wheat long npcRNAs might be regulated by other non-protein coding RNAs, as was the case for Xist gene [44].

\section{Conclusion}

In summary, by using computational analysis and experimental approach, for the first time, we identified 125 putative wheat long npcRNAs. These identified wheat long npcRNAs were not conserved among plant species, and some of them were small RNA precursors. Wheat long npcRNAs showed a tissue dependent expression patterns and their expressions were responsive to powdery mildew infection and/or heat stress, suggesting that they could play roles in development and regulation of biotic and/or abiotic stresses. Our analysis also indicated that expressions of some wheat long npcRNAs could be regulated by small RNAs and through histone acetylation, but this need further investigation. The identification and expression analysis of wheat long npcRNAs in this study would provide a starting point to understand their functions and regulatory mechanisms in the future.

\section{Methods}

\section{Plant materials}

Seeds of powdery mildew susceptible wheat cultivar 'JD8' (designated as $\mathrm{S}$ ) and its near isogenic line carrying a powdery mildew resistance gene Pm30 (designated as R) were planted in 8-10 $\mathrm{cm}$ diameter pots. Seedlings were artificially inoculated when the first leaf was fully expanded, with a local prevalent Blumeria. graminis f. sp. tritici isolate E09. Inoculation was performed by dusting or brushing conidia from neighboring sporulating susceptible seedlings onto the test seedlings. Leaf samples were collected from both genotype at 0 and 12 hrs post inoculation (designated as S-0 h, S-12 h, R-0 h, R-12 h), respectively, and frozen in liquid nitrogen and used for RNA extraction.

For heat stress, heat tolerant genotype 'TAM107' was used in this study. Seeds were surface-sterilized in 1\% sodium hypochlorite for $15 \mathrm{~min}$, rinsed in distilled water, and soaked in dark overnight at room temperature. The germinated seeds were transferred into the pots (25 seedlings per pot) containing vermiculite. The treatments were carried out as described by Qin et al [32]. Leaves were collected at 0 and 1 hour after heat treatment (designated TAM-0 h and TAM-1 h) At the end of heat treatments, the leaves were frozen in the liquid nitrogen immediately, and then stored at $-80^{\circ} \mathrm{C}$ for further use.

\section{Microarray analysis}

Total RNA was extracted using Trizol reagent (Invitrogen) following the manufacture's recommendations. Briefly, mRNA was enriched from $80 \sim 90 \mu \mathrm{g}$ total RNA using the RNeasy Plant Mini Kit (QIAGEN) according to the protocol, and was subsequently reversetranscribed to double stranded cDNA using the GeneChip $^{\circledR}$ Two-Cycle cDNA Synthesis Kit. The biotin labeled cRNA was made using the GeneChip ${ }^{\circledR}$ IVT Labeling Kit (Affymetrix, CA, USA). Twenty micrograms of cRNA samples were fragmented and hybridized for $16 \mathrm{~h}$ at $45^{\circ} \mathrm{C}$ to the Affymetrix Wheat Genome Array (Santa Clara, CA, USA). After washing using the Genechip ${ }^{\circledR}$ Fluidics Station 450, arrays were scanned using the Genechip ${ }^{\circledR} 3000$ Scanner that is located in 
Bioinformatics Center at China Agriculture University (NCBI accession Number: GSE27339 http://www.ncbi. nlm.nih.gov/projects/geo/query/acc.cgi?acc=GSE27339).

\section{Small RNA sequencing}

Small RNA libraries (S-0 h, S-12 h, R-0 h, R-12 h, TAM-0 h, TAM-1 h) preparation and sequencing were performed with Solexa sequencing technology (BGI, Shenzhen, China) as described by Sunkar et al [45]. Automated base calling of the raw sequence and vector removal were performed with PHRED and CROSS MATCH programs [46,47]. Trimmed 3' and 5' adapters sequences, removed RNAs less than 17 nt and polyA, only sequences longer than 17 nt with a unique ID were

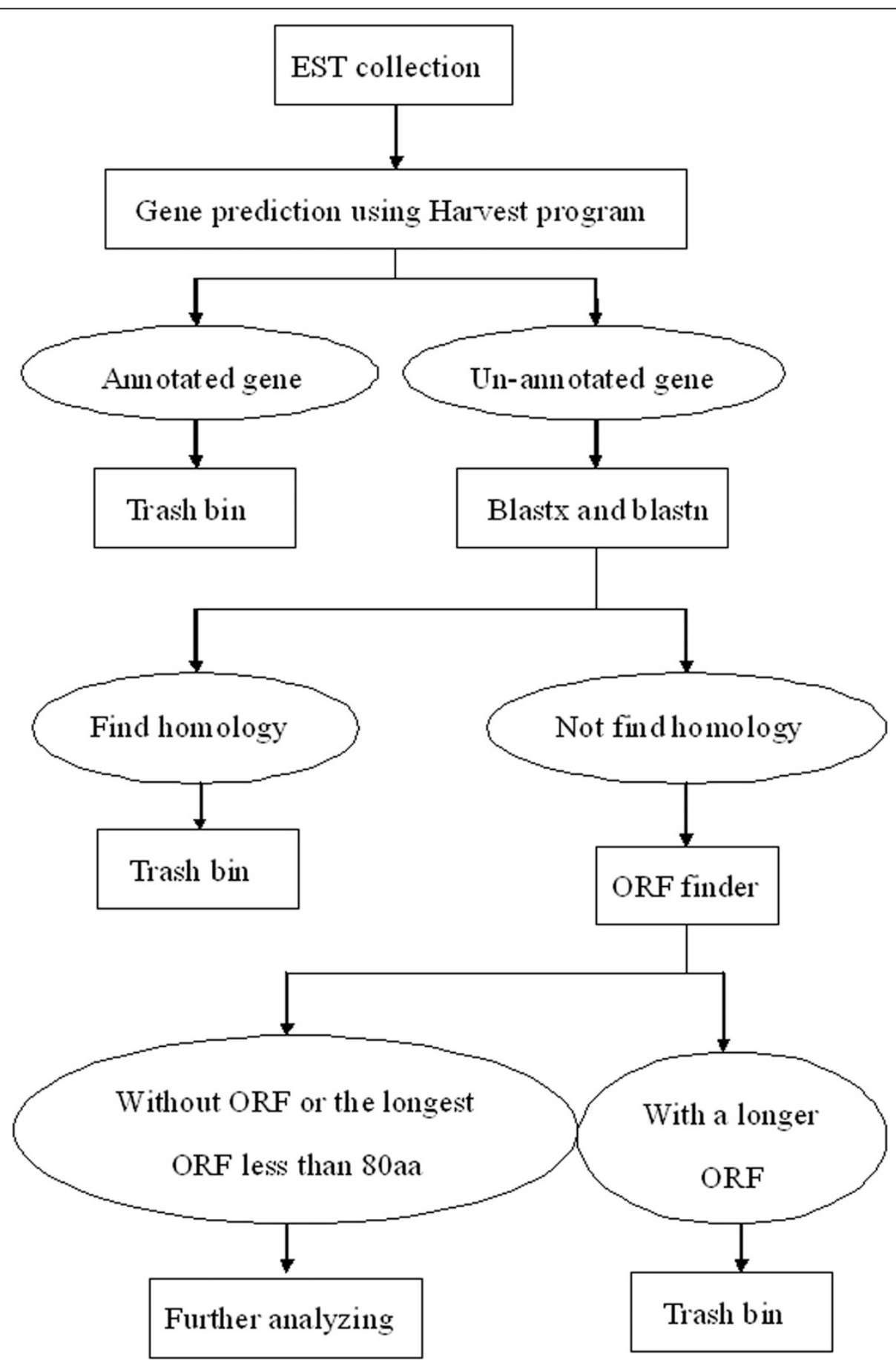

Figure 13 Schematic representation of computational method for long npcRNA identification. 
used for further analysis. We calculated sequencing frequency of each small RNA sequence, the number of reads for each sequence reflecting relative abundance (NCBI accession Number: GSE27339, http://www.ncbi. nlm.nih.gov/projects/geo/query/acc.cgi?acc=GSE27339).

\section{Computational methods for long npcRNA identification}

Firstly, powdery mildew infection and heat stress responsive transcripts were annotated by Harvest program, and protein-coding genes are discarded, the reminding sequences were analyzed by Blastx and Blastn, and ESTs with no similarity to protein coding genes or tRNA and rRNA were retained. Secondly, we screened the reminding ESTs, and the sequences with polyA-tail were selected. Thirdly, we predicted the longest ORF of these transcripts using ORF finder, and the sequences with no or short ORFs (less than 80aa) were retained for further analysis (Figure 13).

\section{Chromatin-Immunoprecipitation (ChIP) assays}

ChIP was modified from published protocol [34]. Approximately $1 \mathrm{~g}$ of leaves was used for each ChIP assay. The fresh tissues were subjected to vacuum infiltration in formaldehyde (1\%) solution for cross-linking the chromatin proteins to DNA. Chromatin was extracted and sonicated for $4 \times 10 \mathrm{sec}$ pulses, $40 \%$ duty cycle and $20 \%$ power with chilling on ice for $1 \mathrm{~min}$ after each pulse. The average size of the resulted DNA fragments ranged between 0.2 2.0$\mathrm{kb}$, centering around $500 \mathrm{bp}$. An aliquot of chromatin solution (1/10 of total volume) was used to determine the DNA fragment sizes and serve as input control. The remaining chromatin solution was diluted 10 -fold and divided into two aliquots. One aliquot was incubated by adding $10 \mu \mathrm{l}$ of antibodies (anti-acetyl-histone H3K9, Upstate Biotechnology, NY). The other aliquot was incubated without antibodies (mock). After incubation at $4^{\circ} \mathrm{C}$ with rotation for overnight, the solution was added to $40 \mu \mathrm{l}$ of protein A agarose and incubated for another 1 hours. The immunocomplexes were eluted and crosslinks were reversed by incubation at $65^{\circ} \mathrm{C}$ for $15 \mathrm{~min}$. Residual protein was degraded by proteinase $\mathrm{K}$ and DNA was extracted and dissolved in $50 \mu \mathrm{l}$ of $\mathrm{dd}_{2} \mathrm{O}$.

\section{Quantitative Real-Time PCR (qRT-PCR) Analysis}

qRT-PCR was performed using the ChIP DNA or cDNA samples in a $10-\mu \mathrm{l}$ mixture containing $1 \times$ LightCyclerFastStart DNA master SYBR Green I. According to MIQE guidelines, qRT-PCR was performed as follows: initial denaturation for $10 \mathrm{~min}$ at $95^{\circ} \mathrm{C}$, followed by 40 cycles of $30 \mathrm{~s}$ at $95^{\circ} \mathrm{C}, 45 \mathrm{~s}$ at 55 to $60^{\circ} \mathrm{C}, 10 \mathrm{~s}$ at $72^{\circ} \mathrm{C}$, and $72^{\circ} \mathrm{C}$ for $5 \mathrm{~min}$ as the last step. The threshold cycles $(\mathrm{Ct})$ of each test target were averaged for triplicate reactions and the values were normalized according to the $\mathrm{Ct}$ of the control products (Ta-actin).

\section{Additional material}

Additional file 1: Wheat long npcRNAs responsive to powdery mildew infection and/or heat stress. The table includes InpcRNAs' ID, probe set ID, the longest ORF, the number of putative ORFs and corresponding siRNAs.

Additional file 2: The hairpin structure of putative TahlnRNA27. The figure shows the secondary structure of putative wheat long npcRNA TahlnRNA27 by using the Vienna RNA package RNAfold web interface program, the perfect hairpin structure indicates that it might give rise to miRNA.

Additional file 3: The hairpin structure of putative TalnRNA5. The figure shows the secondary structure of putative wheat long npcRNA TahlnRNA5 by using the Vienna RNA package RNAfold web interface program, the perfect hairpin structure indicates that it might give rise to miRNA.

Additional file 4: The hairpin structure of putative TalnpmRNA8. The figure shows the secondary structure of putative wheat long npcRNA TahlnRNA8 by using the Vienna RNA package RNAfold web interface program, the perfect hairpin structure indicates that it might give rise to miRNA.

Additional file 5: The hairpin structure of putative TalnpmRNA19. The figure shows the secondary structure of putative wheat long npcRNA TahlnRNA19 by using the Vienna RNA package RNAfold web interface program, the perfect hairpin structure indicates that it might give rise to miRNA.

Additional file 6: Categories of siRNAs corresponding to SRP1 and SRP3 7S RNA variants and sequences of SRP1 and SRP2

corresponding siRNAs. (a) The siRNAs corresponding to SRP1 and SRP3 7S RNA variants are categorized to 5 groups according to their locations, most members of group I, II, and III match both TalnRNA9 and

TalnRNA12, and other two (group IV group V) are specific for TalnRNA9. (b) The table includes sequences of of SRP1 and SRP3 corresponding siRNAs

Additional file 7: Primer sequences for 5'RACE and real time PCR. The table displays the sequences of primers used for both 5'RACE and real time PCR

Additional file 8: The short possible ORFs in TalnRNA21. The figure displays all the possible ORFs in the full length cDNA of TalnRNA21, and none of them are longer than 80aa.

Additional file 9: The short possible ORFs in TahlnRNA37. The figure displays all the possible ORFs in the full length cDNA of TalnRNA37, and none of them are longer than 80aa.

Additional file 10: The short possible ORFs in TahlnRNA47. The figure displays all the possible ORFs in the full length CDNA of TalnRNA47, and none of them are longer than 80aa.

\section{Acknowledgements}

This work was financially supported by National Basic Research Program of China (2007CB109000), 863 Project of China (2007AA10Z138, 2006AA10A104) and National Natural Science Foundation of China (30871529, 30871528).

\section{Author details}

${ }^{1}$ State Key Laboratory for Agrobiotechnology and Key Laboratory of Crop Heterosis and Utilization (MOE) and Key Laboratory of Crop Genomics and Genetic Improvement (MOA), Beijing Key Laboratory of Crop Genetic Improvement, China Agricultural University, Beijing, 100094, PR China. ${ }^{2}$ National Plant Gene Research Centre (Beijing), Beijing 100094, PR China. ${ }^{3}$ Department of Plant Genetics \& Breeding, China Agricultural University, Yuanmingyuan Xi Road No. 2, Haidian District, Beijing, 100193, PR China.

\section{Authors' contributions}

$M X, Y W, Y Y$ and DQ carried out the microarray analysis and small RNA sequencing, participated in the long npcRNA identification, and draft the manuscript. MX and ZH carried out ChIP analysis, NS, CX and HP carried out 
the qRT-PCR analysis. ZN and QS carried out the design of the study and finish the manuscript. All authors read and approved the final manuscript.

\section{Received: 21 October 2010 Accepted: 7 April 2011}

Published: 7 April 2011

\section{References}

1. Ponting $C P$, Oliver $P L$, Reik W: Evolution and functions of long noncoding RNAs. Cell 2009, 136:629-641.

2. Lareau LF, Green RE, Bhatnagar RS, Brenner SE: The evolving roles of alternative splicing. Curr Opin Struct Biol 2004, 14:273-282.

3. Yang XJ: Multisite protein modification and intramolecular signaling. Oncogene 2005, 24:1653-1662.

4. Li L, Wang X, Sasidharan R, Stolc V, Deng W, He H, Korbel J, Chen X, Tongprasit W, Ronald P, Chen R, Gerstein M, Deng XW: Global identification and characterization of transcriptionally active regions in the rice genome. PLoS One 2007, 2:e294.

5. Shukla LI, Chinnusamy V, Sunkar R: The role of microRNAs and other endogenous small RNAs in plant stress responses. Biochim Biophys Acta 2008, 1779:743-748.

6. Lu YD, Gan $Q H$, Chi XY, Qin S: Roles of microRNA in plant defense and virus offense interaction. Plant Cell Rep 2008, 27:1571-1579.

7. Lu S, Sun YH, Amerson H, Chiang VL: MicroRNAs in loblolly pine (Pinus taeda L.) and their association with fusiform rust gall development. Plant J 2007, 51:1077-1098.

8. Navarro L, Dunoyer P, Jay F, Arnold B, Dharmasiri N, Estelle M, Voinnet O, Jones JD: A plant miRNA contributes to antibacterial resistance by repressing auxin signaling. Science 2006, 312:436-439.

9. Sunkar R, Chinnusamy V, Zhu J, Zhu JK: Small RNAs as big players in plant abiotic stress responses and nutrient deprivation. Trends Plant Sci 2007, 12:301-309.

10. Erdmann VA, Szymanski M, Hochberg A, de GN, Barciszewski J: Collection of mRNA-like non-coding RNAs. Nucleic Acids Res 1999, 27:192-195.

11. Okazaki Y, Furuno M, Kasukawa T, Adachi J, Bono H, Kondo S, Nikaido I, Osato N, Saito R, Suzuki H, Yamanaka I, Kiyosawa H, Yagi K, Tomaru Y, Hasegawa Y, Nogami A, Schonbach C, Gojobori T, Baldarelli R, Hill DP, Bult C, Hume DA, Quackenbush J, Schriml LM, Kanapin A, Matsuda H, Batalov S, Beisel KW, Blake JA, Bradt D, Brusic V, Chothia C, Corbani LE, Cousins S, Dalla E, Dragani TA, Fletcher CF, Forrest A, Frazer KS, Gaasterland T, Gariboldi M, Gissi C, Godzik A, Gough J, Grimmond S, Gustincich S, Hirokawa N, Jackson IJ, Jarvis ED, Kanai A, Kawaji H, Kawasawa $Y$, Kedzierski RM, King BL, Konagaya A, Kurochkin IV, Lee $Y$, Lenhard B, Lyons PA, Maglott DR, Maltais L, Marchionni L, McKenzie L, Miki H, Nagashima T, Numata K, Okido T, Pavan WJ, Pertea G, Pesole G, Petrovsky N, Pillai R, Pontius JU, Qi D, Ramachandran S, Ravasi T, Reed JC, Reed DJ, Reid J, Ring BZ, Ringwald M, Sandelin A, Schneider C, Semple CA, Setou M, Shimada K, Sultana R, Takenaka Y, Taylor MS, Teasdale RD, Tomita M, Verardo R, Wagner L, Wahlestedt C, Wang $Y$, Watanabe $Y$, Wells C, Wilming LG, Wynshaw-Boris A, Yanagisawa M, Yang I, Yang L, Yuan Z, Zavolan M, Zhu Y, Zimmer A, Carninci P, Hayatsu N, HirozaneKishikawa T, Konno H, Nakamura M, Sakazume N, Sato K, Shiraki T, Waki K, Kawai J, Aizawa K, Arakawa T, Fukuda S, Hara A, Hashizume W, Imotani K, Ishii Y, Itoh M, Kagawa I, Miyazaki A, Sakai K, Sasaki D, Shibata K, Shinagawa A, Yasunishi A, Yoshino M, Waterston R, Lander ES, Rogers J, Birney $E$, Hayashizaki $Y$ : Analysis of the mouse transcriptome based on functional annotation of 60,770 full-length cDNAs. Nature 2002, 420:563-573.

12. Numata K, Kanai A, Saito R, Kondo S, Adachi J, Wilming LG, Hume DA, Hayashizaki Y, Tomita M: Identification of putative noncoding RNAs among the RIKEN mouse full-length cDNA collection. Genome Res 2003, 13:1301-1306.

13. Ota T, Suzuki Y, Nishikawa T, Otsuki T, Sugiyama T, Irie R, Wakamatsu A, Hayashi K, Sato H, Nagai K, Kimura K, Makita H, Sekine M, Obayashi M, Nishi T, Shibahara T, Tanaka T, Ishii S, Yamamoto J, Saito K, Kawai Y, Isono Y, Nakamura Y, Nagahari K, Murakami K, Yasuda T, Iwayanagi T, Wagatsuma M, Shiratori A, Sudo H, Hosoiri T, Kaku Y, Kodaira H, Kondo H, Sugawara M, Takahashi M, Kanda K, Yokoi T, Furuya T, Kikkawa E, Omura Y, Abe K, Kamihara K, Katsuta N, Sato K, Tanikawa M, Yamazaki M, Ninomiya K, Ishibashi T, Yamashita H, Murakawa K, Fujimori K, Tanai H, Kimata M, Watanabe M, Hiraoka S, Chiba Y, Ishida S, Ono Y, Takiguchi S, Watanabe S, Yosida M, Hotuta T, Kusano J, Kanehori K, Takahashi-Fujii A, Hara H,
Tanase TO, Nomura Y, Togiya S, Komai F, Hara R, Takeuchi K, Arita M, Imose N, Musashino K, Yuuki H, Oshima A, Sasaki N, Aotsuka S, Yoshikawa Y, Matsunawa H, Ichihara T, Shiohata N, Sano S, Moriya S, Momiyama H, Satoh N, Takami S, Terashima Y, Suzuki O, Nakagawa S, Senoh A, Mizoguchi H, Goto Y, Shimizu F, Wakebe H, Hishigaki H, Watanabe T, Sugiyama A, Takemoto M, Kawakami B, Yamazaki M, Watanabe K, Kumagai A, Itakura S, Fukuzumi Y, Fujimori Y, Komiyama M, Tashiro H, Tanigami A, Fujiwara T, Ono T, Yamada K, Fujii Y, Ozaki K, Hirao M, Ohmori Y, Kawabata A, Hikiji T, Kobatake N, Inagaki H, Ikema Y, Okamoto S, Okitani R, Kawakami T, Noguchi S, Itoh T, Shigeta K, Senba T, Matsumura K, Nakajima Y, Mizuno T, Morinaga M, Sasaki M, Togashi T, Oyama M, Hata H, Watanabe M, Komatsu T, Mizushima-Sugano J, Satoh T, Shirai Y, Takahashi Y, Nakagawa K, Okumura K, Nagase T, Nomura N, Kikuchi H, Masuho Y, Yamashita R, Nakai K, Yada T, Nakamura Y, Ohara O, Isogai T, Sugano S: Complete sequencing and characterization of 21,243 fulllength human cDNAs. Nat Genet 2004, 36:40-45.

14. Jones-Rhoades MW, Bartel DP, Bartel B: MicroRNAS and their regulatory roles in plants. Annu Rev Plant Biol 2006, 57:19-53.

15. Prasanth $\mathrm{KV}$, Spector $\mathrm{DL}$ : Eukaryotic regulatory RNAs: an answer to the 'genome complexity' conundrum. Genes Dev 2007, 21:11-42.

16. Mendes Soares LM, Valcarcel J: The expanding transcriptome: the genome as the 'Book of Sand'. EMBO J 2006, 25:923-931.

17. He H, Cai L, Skogerbo G, Deng W, Liu T, Zhu X, Wang Y, Jia D, Zhang Z, Tao Y, Zeng H, Aftab MN, Cui Y, Liu G, Chen R: Profiling Caenorhabditis elegans non-coding RNA expression with a combined microarray. Nucleic Acids Res 2006, 34:2976-2983.

18. Ben AB, Wirth $S$, Merchan $F$, Laporte $P$, ubenton-Carafa $Y$, Hirsch J, Maizel A Mallory A, Lucas A, Deragon JM, Vaucheret H, Thermes C, Crespi M: Novel long non-protein coding RNAs involved in Arabidopsis differentiation and stress responses. Genome Res 2009, 19:57-69.

19. Swiezewski S, Liu F, Magusin A, Dean C: Cold-induced silencing by long antisense transcripts of an Arabidopsis Polycomb target. Nature 2009, 462:799-802.

20. Gupta RA, Shah N, Wang KC, Kim J, Horlings HM, Wong DJ, Tsai MC, Hung T, Argani P, Rinn JL, Wang Y, Brzoska P, Kong B, Li R, West RB, van d, Sukumar S, Chang HY: Long non-coding RNA HOTAIR reprograms chromatin state to promote cancer metastasis. Nature 2010, 464:1071-1076.

21. Rivas E, Eddy SR: Noncoding RNA gene detection using comparative sequence analysis. BMC Bioinformatics 2001, 10:8.

22. Wassarman KM, Repoila F, Rosenow C, Storz G, Gottesman S: Identification of novel small RNAs using comparative genomics and microarrays. Genes Dev 2001, 15:1637-1651.

23. Vercruysse M, Fauvart M, Cloots L, Engelen K, Thijs IM, Marchal K, Michiels J: Genome-wide detection of predicted non-coding RNAs in Rhizobium etli expressed during free-living and host-associated growth using a highresolution tiling array. BMC Genomics 2010, 11:53.

24. Hirsch J, Lefort V, Vankersschaver M, Boualem A, Lucas A, Thermes C, ubenton-Carafa Y, Crespi M: Characterization of 43 non-protein-coding mRNA genes in Arabidopsis, including the MIR162a-derived transcripts. Plant Physiol 2006, 140:1192-1204

25. Inagaki S, Numata K, Kondo T, Tomita M, Yasuda K, Kanai A, Kageyama Y: Identification and expression analysis of putative mRNA-like non-coding RNA in Drosophila. Genes Cells 2005, 10:1163-1173.

26. Maclntosh GC, Wilkerson C, Green PJ: Identification and analysis of Arabidopsis expressed sequence tags characteristic of non-coding RNAs. Plant Physiol 2001, 127:765-776.

27. Gill BS, Appels R, Botha-Oberholster AM, Buell CR, Bennetzen JL, Chalhoub B, Chumley F, Dvorak J, Iwanaga M, Keller B, Li W, McCombie WR, Ogihara Y, Quetier F, Sasaki T: A workshop report on wheat genome sequencing: International Genome Research on Wheat Consortium. Genetics 2004, 168:1087-1096.

28. Griffey CA, Das MK, Stromberg EL: Effectiveness of adult-plant resistance in reducing grain yield loss to powdery mildew in winter wheat. Plant disease 1993, 77:618-622.

29. Wardlaw IF: Interaction between drought and chronic high temperature during kernel filling in wheat in a controlled environment. Ann Bot 2002, 90:469-476.

30. Shukla LI, Chinnusamy V, Sunkar R: The role of microRNAs and other endogenous small RNAs in plant stress responses. Biochim Biophys Acta 2008, 1779:743-748. 
31. Bruggmann $R$, Abderhalden $O$, Reymond P, Dudler R: Analysis of epidermis- and mesophyll-specific transcript accumulation in powdery mildew-inoculated wheat leaves. Plant Mol Biol 2005, 58:247-267.

32. Qin D, Wu H, Peng H, Yao Y, Ni Z, Li Z, Zhou C, Sun Q: Heat stressresponsive transcriptome analysis in heat susceptible and tolerant wheat (Triticum aestivum L.) by using Wheat Genome Array. BMC Genomics 2008, 9:432.

33. Xin MM, Wang Y, Yao YY, Xie CJ, Peng HR, Ni ZF, Sun QX: Diverse set of microRNAs are responsive to powdery mildew infection and heat stress in wheat (Triticum aestivum L.). BMC Plant Biology 2010, 10:123.

34. Lawrence RJ, Earley K, Pontes O, Silva M, Chen ZJ, Neves N, Viegas W, Pikaard CS: A concerted DNA methylation/histone methylation switch regulates rRNA gene dosage control and nucleolar dominance. Mol Cell 2004, 13:599-609.

35. Faghihi MA, Modarresi F, Khalil AM, Wood DE, Sahagan BG, Morgan TE, Finch CE, St LG, Kenny PJ, Wahlestedt C: Expression of a noncoding RNA is elevated in Alzheimer's disease and drives rapid feed-forward regulation of beta-secretase. Nat Med 2008, 14:723-730.

36. Mus E, Hof PR, Tiedge H: Dendritic BC200 RNA in aging and in Alzheimer's disease. Proc Natl Acad Sci USA 2007, 104:10679-10684.

37. Cai $X$, Cullen BR: The imprinted $\mathrm{H} 19$ noncoding RNA is a primary microRNA precursor. RNA 2007, 13:313-316.

38. Tam W: Identification and characterization of human BIC, a gene on chromosome 21 that encodes a noncoding RNA. Gene 2001, 274:157-167.

39. Lu S, Sun YH, Amerson H, Chiang VL: MicroRNAs in loblolly pine (Pinus taeda L.) and their association with fusiform rust gall development. Plant J 2007, 51:1077-1098

40. Navarro L, Dunoyer P, Jay F, Arnold B, Dharmasiri N, Estelle M, Voinnet O, Jones JD: A plant miRNA contributes to antibacterial resistance by repressing auxin signaling. Science 2006, 312:436-439.

41. Li L, Wang X, Sasidharan R, Stolc V, Deng W, He H, Korbel J, Chen X, Tongprasit W, Ronald P, Chen R, Gerstein M, Deng XW: Global identification and characterization of transcriptionally active regions in the rice genome. PLoS One 2007, 2:e294.

42. Shukla LI, Chinnusamy V, Sunkar R: The role of microRNAs and other endogenous small RNAs in plant stress responses. Biochim Biophys Acto 2008, 1779:743-748.

43. Coram TE, Settles ML, Chen XM: Large-scale analysis of antisense transcripts in wheat using Affymetrix GeneChip wheat genome array. BMC genomics 2009, 10:253.

44. Lee JT: Regulation of X-chromosome counting by Tsix and Xite sequences. Science 2005, 309:768-771.

45. Zhang $H$, Yang $J H$, Zheng YS, Zhang $P$, Chen $X, W u J$, Xu L, Luo XQ, Ke ZY, Zhou H, Qu LH, Chen YQ: Genome-wide analysis of small RNA and novel MicroRNA discovery in human acute lymphoblastic leukemia based on extensive sequencing approach. PLoS One 2009, 4:e6849.

46. Sunkar R, Zhu JK: Novel and stress-regulated microRNAs and other small RNAs from Arabidopsis. Plant Cell 2004, 16:2001-2019.

47. Sunkar R, Girke T, Jain PK, Zhu JK: Cloning and characterization of microRNAs from rice. Plant Cell 2005, 17:1397-1411.

doi:10.1186/1471-2229-11-61

Cite this article as: Xin et al:: Identification and characterization of wheat long non-protein coding RNAs responsive to powdery mildew infection and heat stress by using microarray analysis and SBS sequencing. BMC Plant Biology 2011 11:61.

\section{Submit your next manuscript to BioMed Central and take full advantage of:}

- Convenient online submission

- Thorough peer review

- No space constraints or color figure charges

- Immediate publication on acceptance

- Inclusion in PubMed, CAS, Scopus and Google Scholar

- Research which is freely available for redistribution

Submit your manuscript at www.biomedcentral.com/submit
Ciomed Central 